\title{
Hydrostatic pressure effect on mechanical behavior and texture evolution of $\mathrm{Al}$ and Brass
}

Jingyi Zhang ${ }^{1,}$, Karyn Nash ${ }^{1}$, Alyssa Arrigoni ${ }^{1}$, Juan P. Escobedo ${ }^{2}$, Jeffrey N. Florando ${ }^{3}$, David P. Field ${ }^{1}$

${ }^{1}$ School of Mechanical and Materials Engineering, Washington State University, Pullman, WA, 99164

\begin{abstract}
The effect of hydrostatic pressure on shear strength and microstructural evolution of polycrystalline FCC metals was investigated. Hydrostatic pressure of up to 5 GPa was imposed on commercial purity aluminum and $70 / 30$ brass samples using a modified opposed-anvil apparatus (tri-anvil) that allows for measurement of shear strength in thin foil specimens. Similar to the previous investigations made in BCC metals ( $\mathrm{Ta}$ and Mo), the shear strength of FCC metals increases significantly as the pressure rises. At $5 \mathrm{GPa}$, the shear strength of aluminum increased to 8 times its value at atmospheric pressure and 70/30 brass increased by a factor of 2.7. EBSD analysis reveals an evident accumulation of dislocations in all sheared samples, with an approximately 50\% decrease in grain diameter. Texture analysis suggests that, in addition to helping form selectively oriented dislocation walls, hydrostatic pressure also serves as a threshold to select certain favorable orientations in sheared metals. We propose that these hydrostatic pressure effects are intrinsically due to the excess volume associated with the cylindrical strain field of dislocation lines.
\end{abstract}

Keywords: Hydrostatic pressure; FCC; shear strength; EBSD; dislocation; texture. 


\section{Introduction}

The fundamental Tresca and von Mises models $[1,2]$ of classical plasticity theory assume that material strength is independent of pressure and that plastic flow initiates solely from deviatoric stresses. The pressure dependent Steinberg-Guinan model [3] includes the effect of pressure, but only in affecting the value of the shear modulus. The studies of Bridgman [4], Weir [5] and Spitzig [6] on a wide selection of metals show substantial strength increase with increasing pressure. Since that time, much work has been put into designing and constructing a wide variety of apparatuses that can generate high levels of pressure on various forms of matter [7]. The most commonly used device is the diamond anvil cell (DAC) [8-10], which can impose high pressure on specimens. However, the standard DAC poses inconveniences such as the very small sample size ( $30 \mu \mathrm{m} \sim 500 \mu \mathrm{m}$ in diameter) and the development of high deviatoric stresses and stress gradients. Alternatively, an opposed-anvil technique based on procedures established by P.W. Bridgman [4, 11-17] have been employed to assess the influence of pressure on material strength [18-20]. This methodology somewhat resembles the high-pressure torsion (HPT) system which produces highly refined microstructures: a thin disk shaped sample is placed between two hardened steel anvils, then pressure is applied with subsequent torsion about a single axis coinciding with the sample axis [21, 22]. The experiment consists of recording the force required to rotate the anvils as a function of the applied pressure [11-13]. Obviously, the shear deformation imposed on specimens is linearly dependent on the radius position on the axial disk surface $(d=\theta \Delta r)$. To remove this shear deformation gradient introduced by the twisting and disk axes overlapping, a tri-anvil instrument was designed based on the idea of placing specimens away from the twisting axis. This modified instrument is able to isolate hydrostatic pressure effects as it has relatively uniform shear deformation and no pressure gradient over the samples. This feature enables the test to provide a reliable and well controlled procedure to study the effects introduced by pressure without having to deconvolute the effects introduced from the gradient in shear strain. A detailed description of the tri-anvil method can be found in our previous publication [23]. Using the tri-anvil apparatus, a considerable increase in shear strength was measured in BCC Ta 
and Mo specimens subjected to pressures in the 1-5 GPa range [23]. Well-developed dislocation cells were observed both experimentally by TEM study and computationally with dislocation dynamics simulation when the superimposed pressure was high (4 GPa), and the formation of this dislocation network is believed to be the reason for the strengthening effect [17].

The dislocation activity depends upon the available slip systems and is inherently related to the crystalline structure of the given material [24-26]. Consequently, the immediate question that follows is to determine if similar behavior as that observed in BCC Ta would be exhibited in nonBCC metals. To answer this question, experiments were conducted to investigate the effect of pressure on the shear response of FCC metals with different stacking fault energies. Specifically, the results reported herein comprise the shear strength measurements on commercial purity aluminum and $70 / 30$ brass foils. Since it is well established that dislocation glide will lead to the rise of preferred orientation of the crystal, or texture [27], we expect that the unique dislocation motion under pressure will give rise to texture evolution. Post mortem study of the microstructure of these FCC metals was conducted using electron backscatter diffraction (EBSD) analysis [28].

\section{Experimental procedure}

Thin disk-shaped specimens with a diameter of $3.175 \mathrm{~mm}$ were acquired from metal foils using a typical TEM punch. The thickness of the commercial purity (CP) aluminum foil is $26.9( \pm 1.7)$ $\mu \mathrm{m}$, and 77.2 ( \pm 9.1$) \mu \mathrm{m}$ for $70 / 30$ brass. As prepared specimens were then processed with the tri-anvil instrument and deformed to shear strains of approximately 10 under hydrostatic pressures of 3, 4 and $5 \mathrm{GPa}$. The ability of the apparatus to generate shear while superimposing hydrostatic pressure on thin foil samples was verified in our previous publications $[17,23]$. The tri-anvil design uses a similar approach to the Bridgeman single anvil press, which enables hydrostatic pressure and shear loading at the same time. The major modification for the trianvil set-up is that three identical anvil sets are aligned on the periphery of a large circle. The ratio of the radius of the large circle to the radius of the specimen is large enough $(30: 1)$ that 
one can assume a uniform linear displacement between the top and bottom anvils in each set. This displacement is recorded by built-in extensometers mounted directly in each compressing anvil set. The as acquired displacement is then divided by the original foil thickness to generate shear strain results. There are two loading steps in the tests, first a compression is applied to the specimens through the anvil sets to impose pressure and keep the specimens from sliding. Then, as the compressive load is held constant, the bottom anvils rotate a small angle $\left(\theta \approx 0.5^{\circ}\right)$ around the axis of the large circle making an in-plane translational motion relative to the mating top anvil, creating a shear under pressure condition for the thin foil specimens. All specimens, after being sheared under various superimposed pressures along with the original foil samples were then mechanically polished on their axial surface for EBSD observation [29], with a finishing step in the vibratory polisher using $0.02 \mu \mathrm{m}$ colloidal silica suspension.
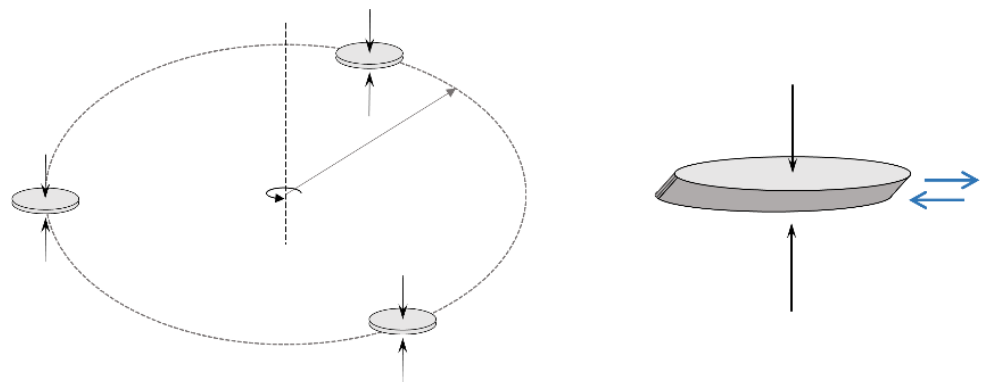

Figure 1 : Illustration of the tri-anvil set up. On the left the three disks represent the location of specimens, the radius of the large circle is $44 \mathrm{~mm}$. On the right shows the deformation state of each specimen under shear loading.

EBSD data of as polished samples were collected with a Schottkey source field emission scanning electron microscope. The scanning step size was kept relatively small with respect to the grain size in each sample: $0.1 \mu \mathrm{m}$ for aluminum and $0.08 \mu \mathrm{m}$ for $70 / 30$ brass. Each sample was scanned over a surface area that contained more than 10,000 grains to generate statistically reliable texture information. EBSD analysis was then carried out using commercial software (TSL OIM Analysis 5). Scan points with low confidence index number $(\mathrm{Cl}<0.1)$ [30] were eliminated. The grain tolerance angle to define grain boundaries was set to $5^{\circ}$. Average $\mathrm{Cl}$ after 
filtering was around 0.8 for all samples, no cleanup or alteration of data was performed for texture analysis.

\section{Results and discussion}

A. Pressure influence on shear strength

Applied axial pressure, torque, and extensometer displacement were recorded by data collection software connected to the tri-anvil apparatus. Torque was then converted to shear stress and plotted against extensometer displacement which was converted to shear strain, generating shear stress/shear strain curves (Figure 2). Both aluminum and 70/30 brass stressstrain curves have notable shear stress increase as a function of rising pressure, and the curves end in an almost linear part with some finite positive slope instead of approaching horizontal (constant stress), this behavior is consistent with the observations made by Bridgman [13] that superimposed hydrostatic pressure generally increases the ductility and strength of metals. It suggests that the strengthening mechanism previously observed in BCC structured metals also applies to FCC metals.
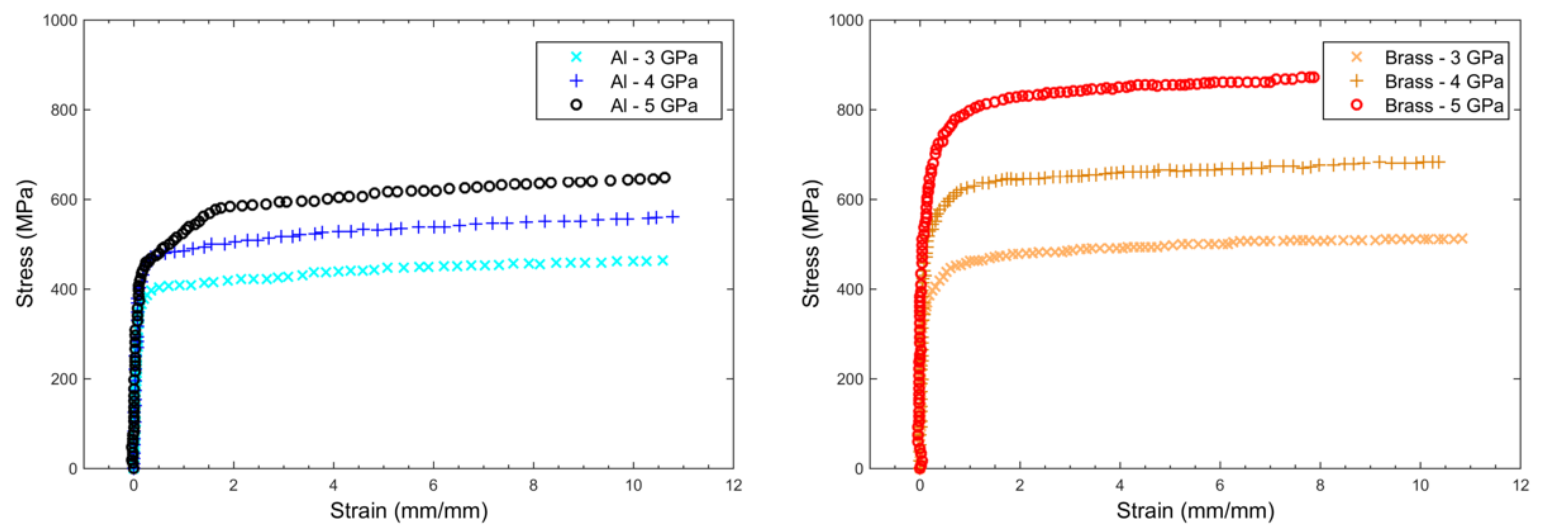

Figure 2: Shear stress - strain curve of CP aluminum (left) and 70/30 brass (right) under 3, 4, and 5 GPa hydrostatic pressures. 
Stress value at strain=1 for each curve was extrapolated as a measure for flow stress (referred to as "shear strength" here and after following Bridgman) and plotted against pressure (Figure 2). Shear strength value in Figure 2 was also normalized with respect to the typical shear strength for each metal at atmospheric pressure and plotted as an inset to show the relative strengthening effect. Both metals show a clear tendency of shear strength increase with rising pressure: Shear strength of aluminum increased to more than 8 times ( $700 \%$ increase) its value at $1 \mathrm{~atm}$ and 70/30 brass increased to about 2.7 times (170\% increase). These scales are in good agreement with Bridgman's calculation in which he concludes 10 times shear strength increase in aluminum, 4.45 times increase for copper and 2 times increase for zinc under $4.9 \mathrm{GPa}$ hydrostatic pressure $[11,13]$.

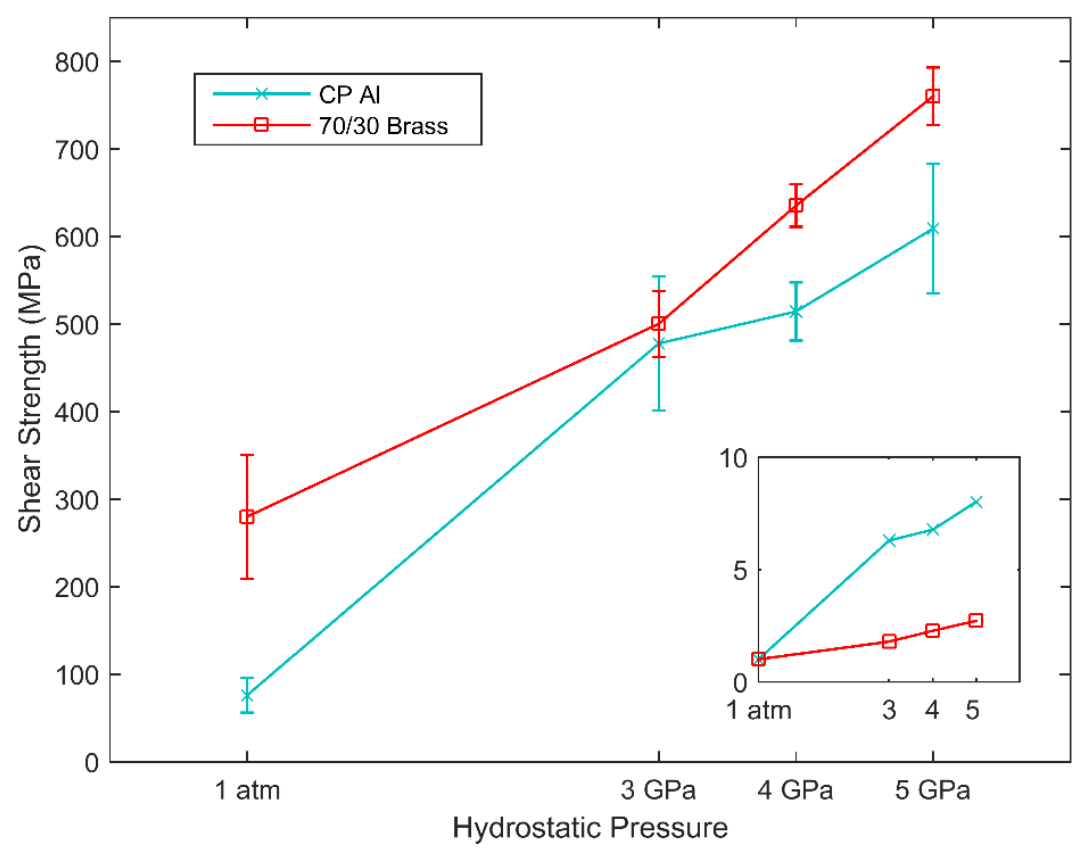

Figure 3: Shear strength of both metals extrapolated from figure 2. Inside: the same value normalized with respect to the shear strength of each metal at $1 \mathrm{~atm}$.

It is natural to consider the strength increase as a result of the refinement of grain size since the deformation procedure is intrinsically the same with high-pressure torsion (HPT) [21] processing of metals, and a major strengthening effect of HPT is from grain size refinement. 
Both metals experienced notable grain refinement, the grain diameters decreased about $40 \%^{\sim}$ $50 \%$ as pressure increased to 5 GPa (Figure 4, left, solid lines) and had submicron grain sizes. To quantify the strengthening effect introduced by grain refinement, we invoke the Hall-Petch relation [31, 32]: $\sigma=\sigma_{0}+k d^{-1 / 2}$ where $k$ is a material dependent constant, and $d$ is the grain diameter. With information of grain size and shear strength at ambient pressure we can invoke this equation (value of $\sigma_{0}$ taken from reference [33] ) to calculate the expected shear strength increase due to grain size reduction: the calculation gives $30.13 \%-39.92 \%$ of shear strength increase for aluminum under various pressures and 5.6\%-29.9\% increase for 70/30 brass (Figure 4 , left, broken lines), both being much smaller than the strengths observed. Average values of grain diameter for each specimen are summarized in Table 1. There are some models that consider the pressure dependency of flow stress of metals, that can also be used to explain the increase in shear flow stress we observed here. The Drucker-Prager yield criterion is a wellestablished model with linear dependency on hydrostatic pressure [19, 34]. The Drucker-Prager yield function has the form:

$$
\sigma_{e f f}=\sigma_{0}\left(\varepsilon_{p l}\right)-a I_{1}
$$

Where $\sigma_{\text {eff }}$ is the effective stress, $\sigma_{0}$ is the pure tension yield strength, $I_{1}$ is the first stress invariant, and $a$ is a material coefficient related to the theoretical cohesive strength (yield strength divided by cohesive strength). Existing calibration for commercial purity aluminum have $a=0.3 \%$, gives about $60 \%$ flow stress increase when $5 \mathrm{GPa}$ hydrostatic pressure is present. This is much smaller compared to the measured $700 \%$ increase. . Another thermodynamic model developed by Jung was successfully applied to explain the pressure dependence of aluminum flow stress by Spitzig and Richmond [35]. The equation considers the amount of work done by pressure due to the volumetric effect from dislocation formation and migration.

$$
\tau=\tau_{0}\left(1-\gamma_{0} p\right)\left(1+\frac{2 p}{G_{0}} \frac{d G}{d p}\right)
$$

Here $\tau$ is the shear stress needed to move a screw dislocation, $\tau_{0}$ is the shear stress at 1 atm, $G_{0}$ is the shear modulus at $1 \mathrm{~atm}$. This expression gave the closest fit to experimentally measured 
flow stress of aluminum under pressure up to $800 \mathrm{MPa}$. Using the same parameters we get a flow stress increase of $75 \%$ for aluminum under 5 GPa pressure, still 10 times smaller than our experimental results. A summary of the predicted flow stress of aluminum by the models mentioned above are plotted together with our experimental results in Figure 4 (Right). The significant difference between calculated results and measured results suggest that all the above discussed effects contributed to the flow stress increase we observe here, but are not sufficient to explain the phenomenon qualitatively. Besides the grain size effect, pressure dependency in classical Drucker-Prager yield surface, and dislocation mobility dependency there are other effects that play a dominant role in the pressure range used in the present study.
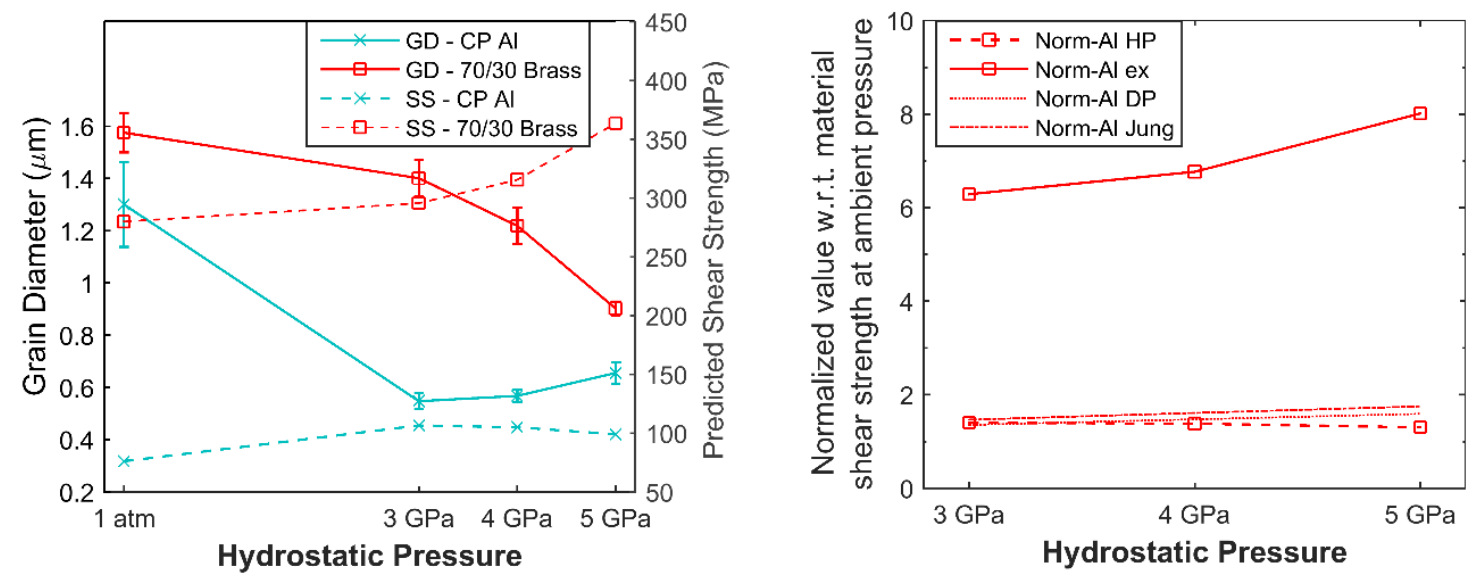

Figure 4 left: Grain diameter (GD) of sheared samples under various hydrostatic pressure (solid lines, left y-axis). And calculated shear strength (SS) using Hall-Petch relation (broken lines, right y-axis). Right: Comparison of $\mathrm{CP}$ aluminum relative flow stress increase predicted by models and experimental data.

\begin{tabular}{lllll}
\hline Unit: $\boldsymbol{\mu m}$ & $\mathbf{1} \mathbf{~ a t m}$ & $\mathbf{3 ~ G P a}$ & $\mathbf{4 ~ G P a}$ & $\mathbf{5 ~ G P a}$ \\
\hline CP Al & $1.301( \pm 0.163)$ & $0.547( \pm 0.030)$ & $0.568( \pm 0.024)$ & $0.656( \pm 0.041)$ \\
\hline 70/30 Brass & $1.576( \pm 0.075)$ & $1.401( \pm 0.069)$ & $1.219( \pm 0.069)$ & $0.902( \pm 0.026)$
\end{tabular}


Table1 Grain diameter summary, units are in microns, all samples but those at 1 atm were sheared to a strain of about 10.

\section{B. Microstructure evolution under pressure}

To better describe and understand the underlying mechanism for the increasing shear strength behavior we observed, texture information on the axial surface of each sample was summarized and studied. Representative orientation maps of original CP aluminum and 70/30 brass foils show that the original 70/30 brass foil (Figure 5 Brass(a)) has a large amount of twinning due to its low stacking fault energy, and the original aluminum foil (Figure $5 \mathrm{Al}(\mathrm{a})$ ) shows a typical rolling microstructure with a high fraction of sub-grains. The crystal lattice orientation within each grain (or sub-grain for aluminum) is almost consistent for both original foils, represented as a uniform color in the orientation map. In contrast, the orientation maps for samples after shearing under pressure (Figure 5 Brass (b) and Al (b)) have color gradients inside the majority of the grains, indicating orientation variation, or accumulation of dislocations. This phenomenon is expected since specimens experienced large deformations in the process. Plastic deformation is intrinsically due to dislocation motion, the shearing step is considered a severe plastic deformation (SPD) procedure which would introduce a large density of dislocations. The dislocation density increase is generally considered to be an important, if not the most important, reason for the work hardening effect from SPD processed metals. In addition to high dislocation density, lath-shaped dislocation cell wall network formation was observed in TEM in BCC Ta samples under higher pressure $[17,23]$. The observation that a lathshaped dislocation network along with dislocation dynamics simulation suggests that this pressure induced dislocation network feature is the potential mechanism for metal strengthening at higher pressures. 

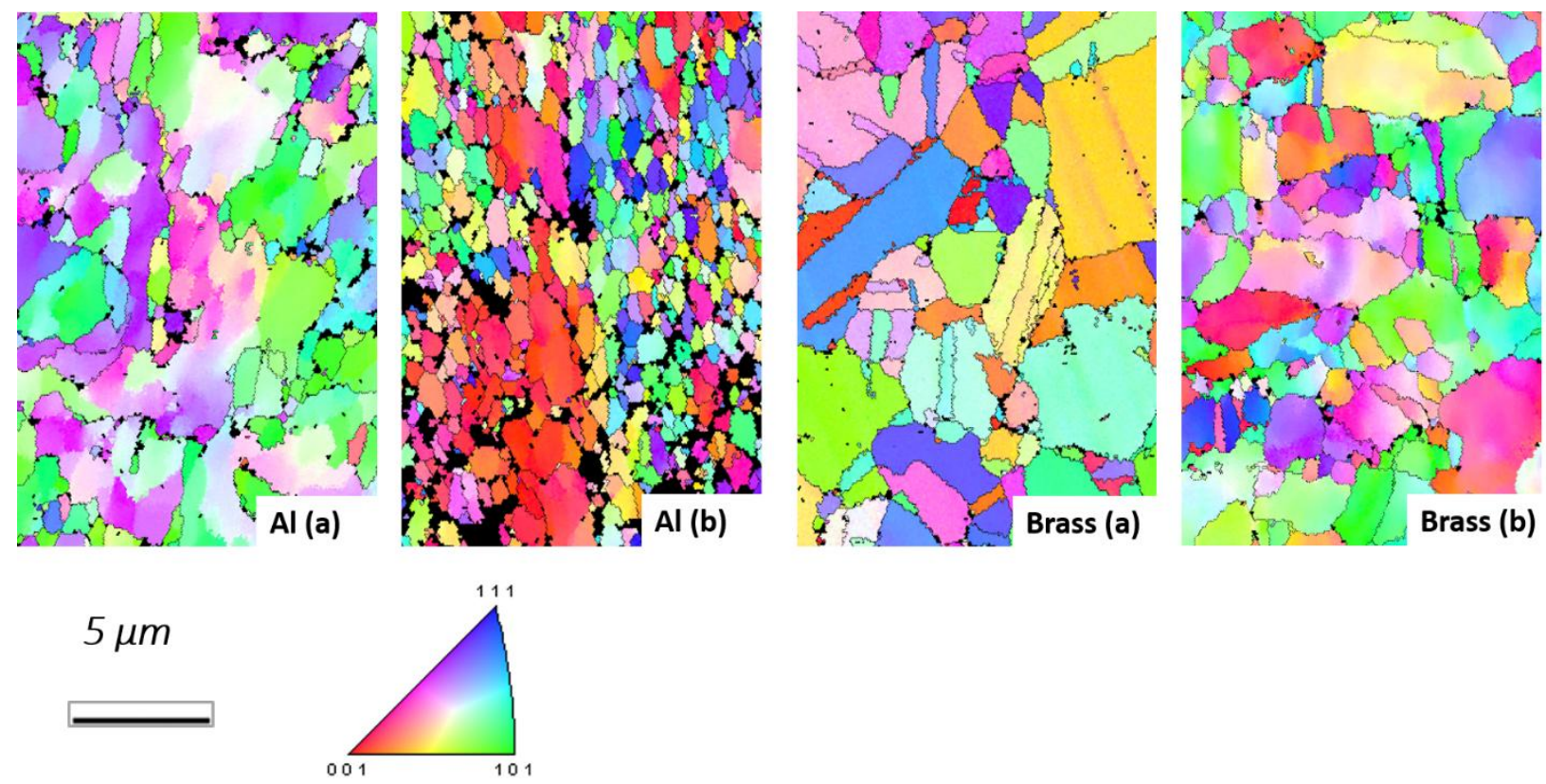

Figure 5: Representative orientation maps of 70/30 brass and CP Aluminum. Original foil orientation maps are indicated with (a), and sheared under 3GPa samples are marked with (b). All figures share the scale bar of $5 \mu \mathrm{m}$ on bottom left, along with color coding for poles normal to the specimen surface shown by the standard triangle.

The same EBSD data were then used to generate pole figures (PF) and orientation distribution functions (ODFs) of all samples for texture evolution analysis. In the $\{111\}$ pole figures both aluminum and 70/30 brass original foils show the typical rolling texture for FCC metals [36, 37]. After the samples were sheared under 3 GPa (Figure 6 - (B), (b)) typical FCC shear texture [37] was formed, confirming the shear deformation during processing. As processing pressure increases, the shear texture holds but is more concentrated to certain orientations with less fiber character. This transition is further studied in the 3D texture representation of ODFs (Figure 7). 


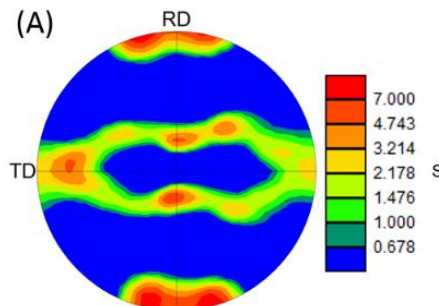

111

(a)

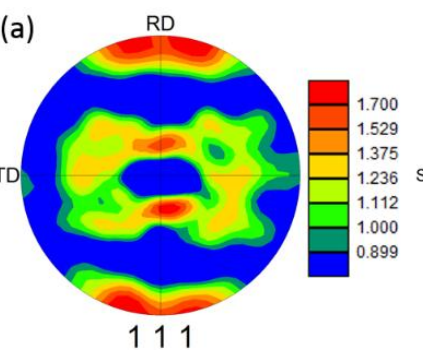

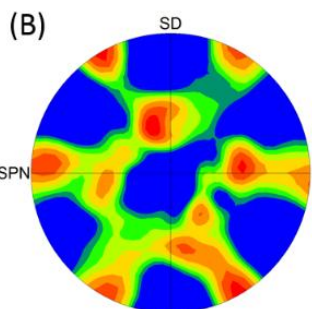

111

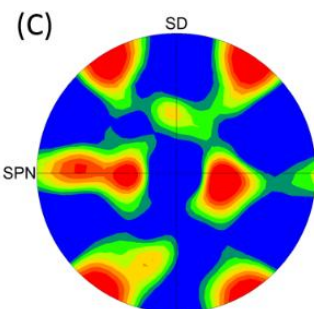

111

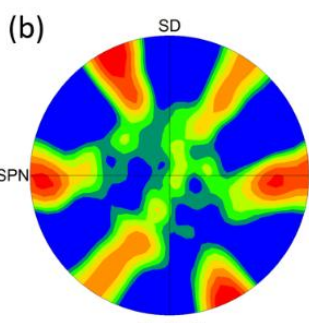

111

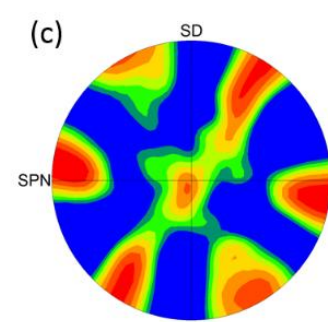

111

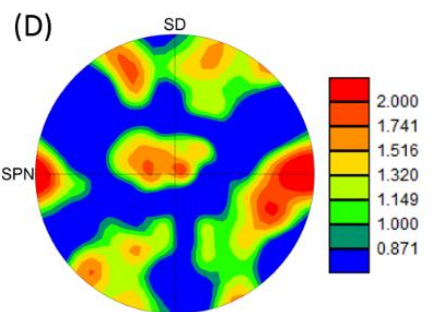

111

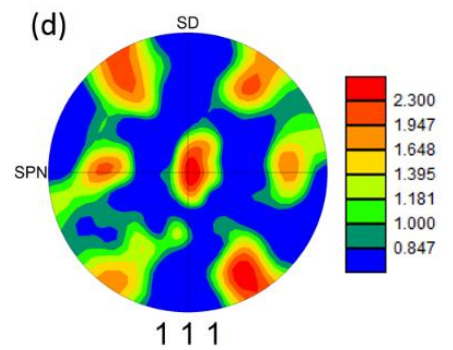

Figure 6: Pole figures of $\{111\}$ direction. Top row: CP aluminum; bottom row: 70/30 brass. (A) and (a) are PFs for the original non- deformed foil samples, (B) and (b) are PFs for samples sheared under 3 GPa, (C) and (c) are for samples sheared under $4 \mathrm{GPa}$, and (D) and (d) are for samples sheared under pressure of 5 GPa. The pole figures for sheared samples of each metal share one common color scale bar. RD and SD are along the vertical axis, and TD and SPN are chosen to be along the horizontal axis.

Within each metal, similar to the texture reported on BCC Ta, the orientation distribution varies for different pressure levels. Firstly, we focus on the second column of the ODF in Figure 7 showing ODFs for sheared 70/30 brass samples under various pressures. A well-defined fiber is formed in the samples sheared at $3 \mathrm{GPa}$, which is the common B-fiber $(\{\mathrm{kh} \mid\}<110>)$ for sheared FCC metals. This confirms that shear deformation occurred rather than sliding of the anvils over the specimens. This B-fiber persists as pressure rises to $4 \mathrm{GPa}$, but it shows less spreading over the $\phi$ angle and becomes a shorter, partial fiber. The resulting partial fiber is further condensed as we increase the superimposed pressure to $5 \mathrm{GPa}$. At $5 \mathrm{GPa}$, the distribution is concentrated into only two peaks (see Figure 6 bottom right), these peaks are the symmetrical positions of the component $\{111\}<110>$. This component is the intersecting point of the $A$-fiber $(\{111\}<u v w>$ and the B-fiber of the ideal shear texture for FCC metal. Aluminum ODFs in the left column for the sheared samples have a similar 'fiber to component' type transition as the pressure 
increases. The aluminum ODF for the $3 \mathrm{GPa}$ sample has a partial $\{001\}<\mathrm{uvw}>$ fiber as well as a partial $\{\mathrm{khl}\}<110>\mathrm{B}$-fiber, and as the pressure increases to $5 \mathrm{GPa}$, the orientation distribution is confined to discrete orientations. In summary, both metals have less orientation spread with increasing pressure. This suggests that hydrostatic pressure has a further "orientation selection" to force the FCC metals to rotate to certain favorable orientations.
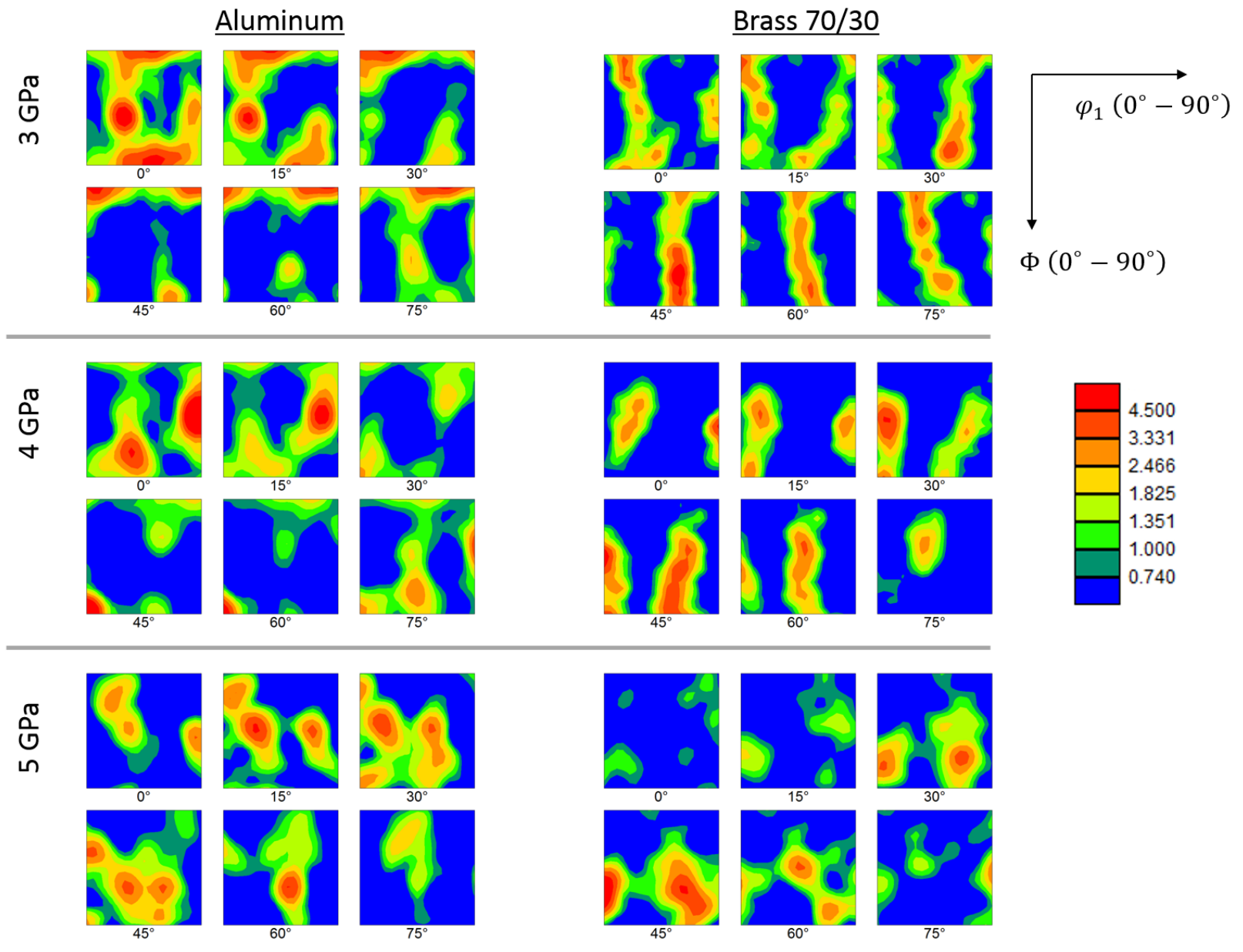

Figure 7: ODFs for CP aluminum samples (left column) and 70/30 brass samples (right column) sheared under 3, 4, and 5 GPa pressure. Each frame shows a constant $\varphi_{2}$ section ranging from $0^{\circ}$ to $75^{\circ}$ with step size of $15^{\circ}$, all frames share the common color scale.

We propose that the crystallographic orientation 'selection' and the formation of lath-shape structured dislocation wall network under higher hydrostatic pressure are two results of the same feature: Dislocations have stress fields that are cylindrically shaped and the affected 
volume is a function of pressure. In particular, edge dislocations are associated with an excessive volume expansion per unit length described by: $\delta v / L=1.0 b^{2}$ where $b$ is the Burgers vector [38]. A multiplication of the $\delta v / L$ term with dislocation density results in the relative volume expansion introduced by a certain density of dislocation in materials is $(\Delta V / V)_{\text {disl }}=\rho_{\text {disl }}(\delta v / L)$. This excess volume feature of dislocation suggests that the formation of unit length of dislocation line, regardless of its spatial direction, would need to overcome an extra energy of approximately $E_{v}=P \cdot \delta v / L$ when the material is under hydrostatic pressure $P$. We can consider $E_{v}$ as a homogenous energy barrier superimposed on samples, with magnitude linearly related to hydrostatic pressure and dislocation density. Some of the dislocations that would have formed under ambient pressure would be impeded by the pressure coupled energy barrier when pressure is in the gigapascal range. In our experiments, only dislocations that strongly accommodate the external shear deformation (that have favorable spatial directions) can overcome the $E_{v}$ barrier under high pressure and result in higher dislocation density along certain directions. The atomistic dislocation study by Bulatov et al [35] on $370 \mathrm{MPa}$ pressure level concluded that the positive (compression) pressure also gives rise to the critical glide stress of screw dislocations by introduction of a volumetric component through transient dilatancy. The static volumetric dilatancy was ruled out as a dominant effect because it introduces too high of a stress increase according to previous experiments under lower pressure values. The fact that our results under higher pressure show large flow stress increases suggests reopening of the discussion of a potential role of the static volumetric change associated with dislocations and other defect structures. Since dislocation movement is intrinsically related to crystallographic texture, the impedance of certain less favorably orientated dislocations as described above is likely to result in the refinement of the crystal orientation distribution spread. The directional selection of dislocations can also explain the formation of previously observed lath-like dislocation cell wall formation, where the majority of dislocations are oriented along similar directions. Further investigation and simulations on dislocation movement is needed to confirm the role of hydrostatic pressure.

\section{Summary and Conclusions}


Shear strength under hydrostatic pressure of FCC materials was investigated in this study. CP aluminum and $70 / 30$ brass thin disk specimens were prepared and sheared with superimposed hydrostatic pressures of 3, 4, and 5 GPa using the Tri-anvil instrument. Shear strengths of both metals increase significantly with increasing pressure in the gigapascal range as is consistent with Bridgman's results $[11,13]$. At 5 GPa pressure, the shear strength of CP aluminum is 8times of its value at atmosphere pressure, and the shear strength of brass increased 2.7 times. Microstructural observations show that both metals have notable grain size reductions (about $50 \%$ at $5 \mathrm{GPa}$ ) after processing, but calculation suggests that the contribution of material strengthening from the grain size effect or dislocation mobility impedance are not enough to explain the enormous increase in shear strength. Further investigation into the textures confirmed that the specimens were under shear deformation. A fiber to component type transition of the ODF is observed in both metals as the pressure increases: after being sheared at $3 \mathrm{GPa}$, the brass has a defined B-fiber, and this fiber gradually reduces into a $\{111\}<110>$ component; aluminum starts with partial A-fiber and B-fiber at $3 \mathrm{GPa}$ and they also transform into discrete components as the pressure increases to $5 \mathrm{GPa}$. We propose that this type of texture refinement is a further orientation selection introduced by hydrostatic pressure and an important mechanism for shear strength enhance of FCC metals as an expense to generate the excess volume for edge dislocations.

\section{Acknowledgement}

This work was performed under the auspices of the U.S. Department of Energy by Lawrence Livermore National Laboratory under Contract DE-AC52-07NA27344.

\section{References}

1. Tresca, H., Mémoire sur l'écoulement des corps solides soumis à de fortes pressions. C.R. Acad. Sci. Paris, 1864. 59: p. 754. 
2. von Mises, R., Mechanik der plastischen Formänderung bei Kristallen. Z. Angew. Math. Mech, 1928. 8(3): p. 161-185.

3. Steinberg, D.J., S.G. Cochran, and M.W. Guinan, A constitutive model for metals applicable at high - strain rate. Journal of Applied Physics, 1980. 51(3): p. 1498-1504.

4. Bridgman, P.W., The Effect of Hydrostatic Pressure on Plastic Flow under Shearing Stress. Journal of Applied Physics, 1946. 17(8): p. 692-698.

5. Weir, S.R., Akella, J., Ruddle, C., Goodwin, T and Siung, L. , Static strength of Ta and $U$ under ultrahigh pressures. Phys. Rev. B, 1998. 57: p. 11258-11265.

6. Spitzig, W.A.a.R., O. , The effect of pressure on the flow stress of metals. Acta Metallurgica, 1984. 32(3): p. 457-463.

7. Lewandowski, J.J. and P. Lowhaphandu, Effects of hydrostatic pressure on mechanical behaviour and deformation processing of materials. International Materials Reviews, 1998. 43(4): p. 145187.

8. Dewaele, A., Loubeyre, P., Mezouar, M., Equations of state of six metals above $94 \mathrm{GPa}$. Physical Review B, 2004. 70(9): p. 094112.

9. Jayaraman, A., Diamond anvil cell and high-pressure physical investigations. Reviews of Modern Physics, 1983. 55(1): p. 65-108.

10. Klepeis, J.-H.P., Cynn, H., Evans, W. J., Rudd, R. E., Yang, L. H., Liermann, H., Yang, W., Diamond anvil cell measurement of high-pressure yield strength of vanadium using in situ thickness determination. Physical Review B, 2010. 81(13): p. 134107.

11. Bridgman, P.W., Effects of High Shearing Stress Combined with High Hydrostatic Pressure. Physical Review, 1935. 48(10): p. 825-847.

12. Bridgman, P.W., Effects of High Hydrostatic Pressure on the Plastic Properties of Metals. Reviews of Modern Physics, 1945. 17(1): p. 3-14.

13. Bridgman, P.W., Flow Phenomena in Heavily Stressed Metals. Journal of Applied Physics, 1937. 8(5): p. 328-336.

14. Bridgman, P.W., On Torsion Combined with Compression. Journal of Applied Physics, 1943. 14(6): p. 273-283.

15. Bridgman, P.W., Explorations toward the Limit of Utilizable Pressures. Journal of Applied Physics, 1941. 12(6): p. 461-469.

16. Bridgman, P.W., The Effect of Pressure on the Tensile Properties of Several Metals and Other Materials. Journal of Applied Physics, 1953. 24(5): p. 560-570.

17. Escobedo, J.P., Field, D. P., Leblanc, M. M., Florando, J. N., Lassila, D. H., Influence of pressure on the microstructural evolution of Ta during shear deformation. Scripta Materialia, 2014. 80(0): $p$. 21-24.

18. Descartes, S., Desrayaud, C., Rauch, E. F., Inhomogeneous microstructural evolution of pure iron during high-pressure torsion. Materials Science and Engineering a-Structural Materials Properties Microstructure and Processing, 2011. 528(10-11): p. 3666-3675.

19. Wilson, C.D., A critical reexamination of classical metal plasticity. Journal of Applied MechanicsTransactions of the Asme, 2002. 69(1): p. 63-68.

20. Kuhlmannwilsdorf, D., Cai, B. C., Nelson, R. B., Plastic-flow between bridgman anvils under highpressures. Journal of Materials Research, 1991. 6(12): p. 2547-2564.

21. Zhilyaev, A.P., Langdon, T. G., Using high-pressure torsion for metal processing: Fundamentals and applications. Progress in Materials Science, 2008. 53(6): p. 893-979.

22. Schafler, E., Strength response upon pressure release after high pressure torsion deformation. Scripta Materialia, 2011. 64(2): p. 130-132. 
23. Escobedo, J.P., Field, D. P., Leblanc, M. M., Florando, J. N., Lassila, D. H., The trianvil test apparatus: Measurement of shear strength under pressure. Review of Scientific Instruments, 2010. 81(1): p. 1-8.

24. Orowan, E., Zur Kristallplastizität. I. Zeitschrift für Physik, 1934. 89: p. 605-634.

25. Taylor, G.I., The Mechanism of Plastic Deformation of Crystals. Part I.-Theoretical. Proceedings of the Physical Society, 1934. A145: p. 362-387.

26. Polanyi, M.Z., Über eine Art Gitterstörung, die einen Kristall plastisch machen könnte. Zeitschrift für Physik, 1934. 89: p. 660-666.

27. Weissmüller, J., Markmann, J., Deforming Nanocrystalline Metals: New Insights, New Puzzles. Adv. Eng. Mater., 2005. 7: p. 202.

28. Schwartz, A.J., et al., Electron backscatter diffraction in materials science. Vol. 2. 2009: Springer New York.

29. Engler, O., Randle, V., Introduction to Texture Analysis: Macrotexture, Microtexture, and Orientation Mapping. 2 ed. 2009: CRC Press. 488.

30. Field, D.P., Recent advances in the application of orientation imaging. Ultramicroscopy, 1997. 67(1-4): p. 1-9.

31. Hall, E.O., The Deformation and Ageing of Mild Steel: III Discussion of Results. Proceedings of the Physical Society. Section B, 1951. 64(9): p. 747.

32. Petch, N.J., The cleavage strength of polycrystals. The Journal of the Iron and Steel Institute, 1953. 173: p. 25-27.

33. Hansen, N., Hall-Petch relation and boundary strengthening. Scripta Materialia, 2004. 51(8): $p$. 801-806.

34. Drucker, D.C. and W. Prager, SOIL MECHANICS AND PLASTIC ANALYSIS OR LIMIT DESIGN. Quarterly of Applied Mathematics, 1952. 10(2): p. 157-165.

35. Bulatov, V.V., O. Richmond, and M.V. Glazov, An atomistic dislocation mechanism of pressuredependent plastic flow in aluminum. Acta Materialia, 1999. 47(12): p. 3507-3514.

36. Stephen, A.W., Texture and Mechanical Anisotropy in the Copper-Zinc System. 1968, University of Arizona.

37. Kocks, U.F., Tomé, C.N., Wenk, H.R., Texture and Anisotropy: Preferred Orientations in Polycrystals and Their Effect on Materials Properties. 2000: Cambridge University Press.

38. Hirth, J.P., Lothe, J Theory of dislocations. 1982, New York: Krieger Publishing Company. 